\title{
Pre-pregnancy Body Mass Index and Third-Trimester Depressive Symptoms in a Healthy Privately Insured Sample
}

\author{
Stephanie A. Schuette, BA ${ }^{1}$ \\ Suena $\mathrm{H}$. Massey, $\mathrm{MD}^{3,4}$ \\ ${ }^{1}$ Department of Psychology and Neuroscience, Duke University,
Durham, North Carolina
2 Department of Obstetrics and Gynecology, Northwestern University
Feinberg School of Medicine, Chicago, Illinois
${ }^{3}$ Department of Psychiatry and Behavioral Sciences, Northwestern
University Feinberg School of Medicine, Chicago, Illinois
${ }^{4}$ Department of Medical Social Sciences, Northwestern University
Feinberg School of Medicine, Chicago, Illinois
}

Michelle A. Kominiarek, MD, MS²

Katherine L. Wisner, MD, MS²,3

Address for correspondence Stephanie A. Schuette, BA, Department of Psychology and Neuroscience, Duke University, 2213 Elba Street, Durham, NC 27705 (e-mail: stephanie.schuette@duke.edu).

Am J Perinatol Rep 2018;8:e13-e17.

\begin{abstract}
Objectives While being overweight (body mass index [BMI] $>25$ ) prior to pregnancy is linked to antenatal depression, whether weight is confounded by socioeconomic and/or medical risks is unclear.

Study Design We assessed 66 healthy privately insured pregnant women at $M=35.0$ \pm 3.3 weeks for symptoms of depression (Inventory for Depressive Symptoms-SelfReport (IDS-SR 30), lifetime history of depression and other psychiatric conditions (Mini International Neuropsychiatric Interview), and pre-pregnancy BMI derived from prepregnancy weight (by recall) and directly measured height. Pre-pregnancy overweight (BMI > 25) and antenatal depression (score of mild or greater [14] on the IDS-SR 30) were assessed using logistic regression, controlling for past major depressive disorder (MDD) and

Keywords

- oxytocin

- overweight

- pregnancy

- postpartum depression demographic factors showing significant between group differences.

Results Pre-pregnancy overweight $(n=17 ; 25.8 \%)$ was associated with an increased risk of third trimester depression, independent of past MDD and marital status (odds ratio $=7.47$; 95\% confidence interval [2.09-26.68]; $B$ (standard error) $=2.010$ [0.650]). Conclusion Replication in a larger sample is suggested to confirm an independent effect of pregravid overweight on third trimester depression.
\end{abstract}

The prevalence of overweight or obesity, defined as a body mass index (BMI) of 25 to 30 , or $>30 \mathrm{~kg} / \mathrm{m}^{2}$, respectively, among women who conceive in the United States is significant and rapidly rising. One-third of women are overweight at the time of conception, and one in five are obese. ${ }^{1}$ Elevated pre-pregnancy BMI has been linked to a variety of adverse obstetric outcomes including but not limited to preeclampsia, hypertension, gestational diabetes, and birth defects. ${ }^{2-4}$

received

June 23, 2017

accepted after revision

December 9, 2017
DOI https://doi.org/

10.1055/s-0038-1625974. ISSN 2157-6998.
Pre-pregnancy overweight and obesity also increase the risk of another common, but less studied pregnancy complication -antenatal depression, or the onset of depressive symptoms in women while pregnant. ${ }^{5}$ There is increasing attention to the identification and treatment of antenatal depression ${ }^{6}$ due in part to a growing recognition of the short- and long-term impact of intrauterine exposure to maternal depression on child outcomes. ${ }^{7}$ Within this context, the
Copyright $\odot 2018$ by Thieme Medical Publishers, Inc., 333 Seventh Avenue, New York, NY 10001, USA. Tel: +1(212) 584-4662.
License terms

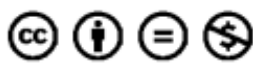


identification of modifiable risk factors for antenatal depression could enable early detection and even prevention of this common complication of child-bearing.

Overweight is a well-established risk factor for depression in nonpregnant individuals. In pregnant women, elevated pre-pregnancy BMI appears to increase the risk of depression illness in a dose-dependent fashion. ${ }^{8}$ However, the link between pre-pregnancy BMI and depression in pregnancy could have etiologic contributions from weight-related nonpsychiatric illnesses and socioeconomic status (SES), ${ }^{9}$ associated with both obesity and depression. ${ }^{10}$ To isolate whether pregravid overweight predicts antenatal depressive symptoms independent of SES and weight-related illnesses (diabetes, hypertension, heart disease), we analyzed data from an existing cohort of healthy, privately-insured pregnant women recruited from a large urban academic medical center. Specifically, we examined the relationship between pre-pregnancy overweight and third-trimester depressive symptoms. We secondarily examined (1) whether depressive symptoms were related to gestational weight gain and (2) the specific depressive symptoms that were most strongly associated with pre-pregnancy weight.

\section{Materials and Methods}

\section{Sample}

Participants were 66 predominantly married, privately insured, pregnant women who were taking part in a longitudinal study about third-trimester plasma oxytocin and postpartum depression. Data for the current study include information collected during initial screening and thirdtrimester visits $(\mathrm{M}=35.0$ weeks; $\mathrm{SD}=3.3)$. Inclusion criteria were age of 18 years or older, English literacy, and third-trimester singleton pregnancy. As the parent study aimed to replicate and expand on previous work on oxytocin and perinatal mood, ${ }^{11}$ potential participants with a BMI $>32$ were excluded. Additional exclusion criteria were (1) any medical illness prior to or during pregnancy including diabetes, hypertension, sleep apnea, or thyroid disease; (2) any current treatment for depression or anxiety (either with antidepressants or psychotherapy); (3) fetal malformation; and (4) cigarette smoking beyond the 10th week of gestation.

\section{Recruitment and Procedures}

A research assistant recruited participants from the waiting area of the academic faculty obstetric clinic that serves exclusively privately insured patients by assessing interest in "a study about oxytocin and mood." Brochures, online advertisements, and flyers were also placed throughout the medical center, affiliated clinics, and in local businesses close to the medical campus. Women who met inclusion and exclusion criteria through initial screening (by phone, for participants responding to advertisements, and in person, for those recruited from the clinic) were invited for baseline assessments.

At screening, pre-pregnancy weight was obtained via selfreport from participants. Current height and weight were measured directly by a research nurse. Current depressive symptoms were assessed using the Inventory for Depressive Symptoms-Self-Report 30 (IDS-SR 30) ${ }^{12}$ which is categorized according to severity: 0 to $13=$ normal; 14 to $25=$ mild depression, 26 to $38=$ moderate depression; 39 to $48=$ severe depression, and 49 to $84=$ very severe depression. ${ }^{13}$ The IDS-SR has been previously validated for use in assessing depression among pregnant women. ${ }^{14}$ Due to the importance of early detection of perinatal depression, we focused on the presence of any depression, however, mild. Thus, we utilized a score of 14 or higher to define the presence of depressive symptoms during pregnancy. The Mini International Neuropsychiatric Interview Version 5.0.0. (MINI) ${ }^{15}$ was administered by study psychiatrists to determine lifetime history of major depressive episodes and other psychiatric conditions. All procedures were approved by the Institutional Review Board of the university where this study was conducted.

\section{Analysis}

BMI was calculated from measured height and pre-pregnancy weight $\left(\mathrm{BMI}=\right.$ weight $[\mathrm{kg}] /$ height $\left.\left[\mathrm{m}^{2}\right]\right)$. A binary variable was created to reflect pre-pregnancy overweight status, defined as a BMI $\geq 25$ (U.S. Department of Health and Human Services, 2015). Based on an IDS-SR 30 score of $\geq 14$, a binary variable was created to reflect the presence of depressive symptoms. Gestational weight gain through the third trimester was calculated by subtracting pre-pregnancy weight from weight measured at the third-trimester visit. Demographic and clinical characteristics were compared between pre-pregnancy overweight and pre-pregnancy not overweight groups using $t$-tests and chi-square tests for continuous and binary variables, respectively. The association between pre-pregnancy overweight (independent variable) and third-trimester depressive symptoms (dependent variable) was assessed using logistic regression analysis, with past major depressive disorder (MDD) (as determined by the MINI) entered as a covariate. The presence of multicollinearity among factors was ruled out using bivariate correlation analysis. Finally, relationships among pre-pregnancy BMI, gestational weight gain, and specific depressive symptoms reported in the IDS-SR 30 were explored using bivariate correlation analysis.

\section{Results}

Racial and ethnic breakdown were non-Hispanic Caucasian 72.7\% $(n=48)$; African American 10.6\% $(n=7)$; Asian 9.1\% $(n=6)$; and Hispanic $7.6 \%(n=5)$. The distribution of prepregnancy BMI in this sample was underweight (BMI $<18.5$ ) $=4.5 \%(n=3)$; normal weight (BMI 18.5-24.9) $=68.2 \%$ $(n=46)$; overweight (BMI 25-29.9) $=16.7 \%(n=11)$; and obese $(\mathrm{BMI} \geq 30-32)=10.6 \%(n=6)$. One in five participants $(n=13)$ had a prior major depressive episode (MINI); the length of time between prior episode and the current pregnancy ranged from 2 to 12 years. Other past psychiatric diagnoses were panic disorder $(n=2)$, posttraumatic stress disorder $(n=1)$, obsessive-compulsive disorder $(n=1)$, 
Table 1 Characteristics of participants by pregravid overweight status (BMI $\geq 25$ )

\begin{tabular}{|l|l|l|l|}
\hline & Total $(\boldsymbol{N}=\mathbf{6 6})$ & Not overweight $(\boldsymbol{n}=\mathbf{4 9})$ & Overweight $(\boldsymbol{n}=\mathbf{1 7})$ \\
\hline Age $(\mathrm{y})$ & $33.0(4.9)$ & $33.0(4.4)$ & $33.0(6.2)$ \\
\hline Gestational age $(\mathrm{wk})$ & $35.0(3.3)$ & $34.8(3.3)$ & $35.7(3.3)$ \\
\hline Unmarried & $12.1 \%$ & $6.1 \%^{\mathrm{a}}$ & $23.5 \%^{\mathrm{a}}$ \\
\hline Multiparous & $55.4 \%$ & $63.4 \%$ & $41.2 \%$ \\
\hline Racial/ethnic minority & $30.3 \%$ & $26.5 \%$ & $41.2 \%$ \\
\hline Pregravid BMI $\left(\mathrm{kg} / \mathrm{m}^{2}\right)$ & $23.2(3.7)$ & $21.3(1.8)^{\mathrm{b}}$ & $28.5(2.2)^{\mathrm{b}}$ \\
\hline Weight gained to date (lbs) & $30.0(10.1)$ & $30.8(9.7)$ & $29.8(12.4)$ \\
\hline Current BMI $\left(\mathrm{kg} / \mathrm{m}^{2}\right)$ & $28.3(4.2)$ & $26.3(2.5)^{\mathrm{b}}$ & $33.9(2.8)^{\mathrm{b}}$ \\
\hline Lifetime MDD & $20.0 \%$ & $22.4 \%$ & $11.8 \%$ \\
\hline Lifetime psych dx $^{\mathrm{c}}$ & $29.2 \%$ & $30.6 \%$ & $17.6 \%$ \\
\hline Current depression $^{\mathrm{d}}$ & $34.8 \%$ & $24.5 \%^{\mathrm{b}}$ & $64.7 \%^{\mathrm{b}}$ \\
\hline
\end{tabular}

Abbreviations: BMI, body mass index; MDD, major depressive disorder.

${ }^{\mathrm{a}} p=<0.05$.

${ }^{\mathrm{b}} p=<0.01$.

${ }^{c}$ Mini International Neuropsychiatric Interview Version 5.0.0 regarding history prior to pregnancy.

Inventory of Depressive Symptoms-Self-Report 30 item version score $\geq 14$ suggestive of least mild depression.

generalized anxiety disorder $(n=1)$, alcohol abuse $(n=1)$, alcohol dependence $(n=1)$, marijuana abuse $(n=1)$, and antisocial personality disorder $(n=1)$. Descriptive characteristics of pre-pregnancy overweight/obesity and normal weight women are shown in -Table 1. Overweight/obese women were significantly more likely to be unmarried.

Prior major depression and pre-pregnancy overweight status were associated with third-trimester depression (-Table 2) and together accounted for a quarter of the variance (Nagelkerke's $R^{2}=0.248$ ). Pre-pregnancy overweight/obesity was associated with an approximately sevenfold increase in the likelihood of third-trimester depression. Gestational weight gain was unrelated to third-trimester depressive symptoms $(r=-0.009, p=0.953)$. Pre-pregnancy overweight/obesity was significantly correlated with the IDS-SR 30 item, "waking up too early" $(r=0.267$, $p=0.030$ ) but not with other items.

\section{Discussion}

Although overweight and depression are more prevalent among socioeconomically disadvantaged women, ${ }^{16}$ there is recent evidence that the relationship between BMI and antenatal depression occurring during gestation is stronger among women with higher SES. ${ }^{17}$ In this study, we present preliminary evidence between pre-pregnancy overweight and depressive symptoms in the third trimester among predominantly high SES pregnant women without any medical comorbidities. This relationship held after controlling for lifetime depressive disorder, which itself, conferred a fourfold increase in risk for antenatal depression consistent with studies in much larger samples. ${ }^{18}$ There are numerous mechanisms linking obesity to depression including but not limited to inflammation, ${ }^{19-21}$ hormonal dysregulation, ${ }^{22}$ sleep disturbance, ${ }^{23}$ and psychosocial factors such as body dissatisfaction, particularly among women. ${ }^{24}$ We noted a significant correlation between endorsement of insomnia (early wakening) on the IDS-SR 30 and depression. While this is by no means a formal measure of sleep disturbance, significant overlaps in sleep disturbance, obesity, and depression are well known. ${ }^{25,26}$ Understanding how these factors interact during pregnancy in a prospective study would be informative to identify key targets for preventive interventions.

\section{Limitations}

As is common for secondary analysis studies, there are several limitations that threaten to bias our findings. These include reliance of self-reports of pre-pregnancy weight,

Table 2 Logistic regression model of factors associated with third-trimester depressive symptoms ${ }^{\mathrm{a}}$

\begin{tabular}{|c|c|c|c|c|c|c|c|}
\hline & $B$ & SE & Wald & df & Sig & OR & $95 \% \mathrm{Cl}$ for $\mathrm{OR}$ \\
\hline Unmarried & -0.001 & 0.952 & 0.000 & 1 & 1.000 & 0.999 & $0.155-6.460$ \\
\hline Prior $\mathrm{MDD}^{\mathrm{a}}$ & 1.444 & 0.705 & 4.193 & 1 & 0.041 & 4.239 & $1.064-16.893$ \\
\hline Pregravid overweight/obesity ${ }^{b}$ & 2.010 & 0.670 & 9.006 & 1 & 0.003 & 7.467 & $2.009-27.758$ \\
\hline
\end{tabular}

Abbreviations: $\mathrm{Cl}$, confidence interval; MDD, major depressive disorder; OR, odds ratio; SE, standard error.

aPast MDD, determined by the Mini International Neuropsychiatric Interview Version 5.0.0.

${ }^{\mathrm{b}}$ Defined as pregravid body mass index of $\geq 25$. 
restriction of the sample to women with a BMI of less than 32 , and an elevated prevalence of past MDD (20\%) and any past psychiatric condition (29.2\%) in this sample. However, each of these limitations would be expected to reduce, not magnify the strength of the relationship we found. Specifically, women who were overweight prior to pregnancy would be expected to underestimate their pre-pregnancy weight, thereby restricting the variance in prepregnancy BMI. Similarly, restriction of BMI to $<32$ (required for a study on plasma oxytocin) should further reduce variance in pre-pregnancy BMI. Even with this limited range, we detected a robust effect of pre-pregnancy overweight on prenatal depressive symptoms. Finally, we considered possible confounding of the pre-pregnancy overweight-antenatal depression link by past MDD. However, as shown in - Table 1, the prevalence of past MDD and past psychiatric disorders was actually higher in women who were not overweight before pregnancy. In these ways, we have reason to believe in the validity of our results.

There are also alternative explanations for the relationship between pre-pregnancy overweight and antenatal depression. We considered that bodily discomfort in the third trimester could be more prominent in women who gained a large amount of weight during pregnancy. However, as shown in -Table 1, the amount of weight gained during pregnancy between groups was nearly identical. A further post hoc analysis of pre-pregnancy BMI and IDS-SR 30 items reflecting somatic discomfort (heaviness, pain, lethargy) did not reveal significant correlations. Finally, the relationship found in this study between pre-pregnancy overweight and antenatal depression is higher than reported in existing studies of women with similar SES. ${ }^{17}$ Thus, our sample may not be representative and could reflect the unique attributes of women who seek obstetric care at an academic medical center located in a large city.

\section{Conclusion}

Excessive weight prior to pregnancy in the overweight to mild obesity range may be an independent risk factor for depressive symptoms during pregnancy, independent of SES, weight-related illnesses, and past depressive episodes. Replication of these results in a larger sample would be important in light of potential implications for prevention.

\section{Funding}

This work was supported by a grant from the Evergreen Invitational Women's Health Grants Initiative of the Northwestern Memorial Foundation (NMF) to Dr. Massey, grant 5K23DA037913 from the National Institute on Drug Abuse (NIDA) of the National Institutes of Health (NIH) to Dr. Massey, and grant K23HD076010 from the Eunice Kennedy Shriver National Institute of Child Health \& Human Development (NICHD) of the NIH to Dr. Kominiarek. The NMF, NIDA, NICHD, and NIH had no role in the study design, collection, analysis or interpretation of data, writing of the article, or the decision to submit this article for publication.

\section{Conflict of Interest}

The Department of Psychiatry at Northwestern University receives contractual fees for Dr. Wisner's consultation to Quinn Emanuel Urquhart \& Sullivan, LLP (New York City), who represent Pfizer Pharmaceutical Company.

\section{References}

1 Fisher SC, Kim SY, Sharma AJ, Rochat R, Morrow B. Is obesity still increasing among pregnant women? Prepregnancy obesity trends in 20 states, 2003-2009. Prev Med 2013;56(06): 372-378

2 Ramachenderan J, Bradford J, McLean M. Maternal obesity and pregnancy complications: a review. Aust N Z J Obstet Gynaecol 2008;48(03):228-235

3 Watkins ML, Rasmussen SA, Honein MA, Botto LD, Moore CA. Maternal obesity and risk for birth defects. Pediatrics 2003;111(5 Pt 2):1152-1158

4 Olivarez SA, Ferres M, Antony K, et al. Obstructive sleep apnea screening in pregnancy, perinatal outcomes, and impact of maternal obesity. Am J Perinatol 2011;28(08):651-658

5 Bodnar LM, Wisner KL, Moses-Kolko E, Sit DK, Hanusa BH. Prepregnancy body mass index, gestational weight gain, and the likelihood of major depressive disorder during pregnancy. J Clin Psychiatry 2009;70(09):1290-1296

6 Ashley JM, Harper BD, Arms-Chavez CJ, LoBello SG. Estimated prevalence of antenatal depression in the US population. Arch Women Ment Health 2016;19(02):395-400

7 Gentile S. Untreated depression during pregnancy: short- and long-term effects in offspring. A systematic review. Neuroscience 2017;342:154-166

8 Molyneaux E, Poston L, Ashurst-Williams S, Howard LM. Prepregnancy obesity and mental disorders during pregnancy and postpartum: a systematic review and meta-analysis. Pregnancy Hypertens 2014;4(03):236

9 Bombard JM, Dietz PM, Galavotti C, et al. Chronic diseases and related risk factors among low-income mothers. Matern Child Health J 2012;16(01):60-71

10 Lancaster CA, Gold KJ, Flynn HA, Yoo H, Marcus SM, Davis MM. Risk factors for depressive symptoms during pregnancy: a systematic review. Am J Obstet Gynecol 2010;202(01): 5-14

11 Skrundz M, Bolten M, Nast I, Hellhammer DH, Meinlschmidt G. Plasma oxytocin concentration during pregnancy is associated with development of postpartum depression. Neuropsychopharmacology 2011;36(09):1886-1893

12 Rush AJ, Gullion CM, Basco MR, Jarrett RB, Trivedi MH. The Inventory of Depressive Symptomatology (IDS): psychometric properties. Psychol Med 1996;26(03):477-486

13 Rush AJ, Blacker D. Handbook of Psychiatric Measures. Washington, DC: American Psychiatric Publishing; 2008

14 Brunoni AR, Benute GR, Fráguas R, et al. The self-rated Inventory of Depressive Symptomatology for screening prenatal depression. Int J Gynaecol Obstet 2013;121(03):243-246

15 Sheehan D, Janavs J, Knapp E, Sheehan M, Baker R. MiniInternational Neuropsychiatric Interview Clinician Rated, Version 4.0. Tampa, FL: University of South Florida College of Medicine; 1992

16 Luppino FS, de Wit LM, Bouvy PF, et al. Overweight, obesity, and depression: a systematic review and meta-analysis of longitudinal studies. Arch Gen Psychiatry 2010;67(03):220-229

17 Molyneaux E, Pasupathy D, Kenny LC, et al; SCOPE consortium. Socio-economic status influences the relationship between obesity and antenatal depression: data from a prospective cohort study. J Affect Disord 2016;202:124-127

18 Rich-Edwards JW, Kleinman K, Abrams A, et al. Sociodemographic predictors of antenatal and postpartum depressive symptoms 
among women in a medical group practice. J Epidemiol Community Health 2006;60(03):221-227

19 Castanon N, Lasselin J, Capuron L. Neuropsychiatric comorbidity in obesity: role of inflammatory processes. Front Endocrinol (Lausanne) 2014;5:74

20 Molica F, Morel S, Kwak BR, Rohner-Jeanrenaud F, Steffens S. Adipokines at the crossroad between obesity and cardiovascular disease. Thromb Haemost 2015;113(03):553-566

21 Quante M, Dietrich A, ElKhal A, Tullius SG. Obesity-related immune responses and their impact on surgical outcomes. Int J Obes 2015;39(06):877-883

22 Joseph JJ, Golden SH. Cortisol dysregulation: the bidirectional link between stress, depression, and type 2 diabetes mellitus. Ann N Y Acad Sci 2017;1391(01):20-34
23 Broussard JL, Van Cauter E. Disturbances of sleep and circadian rhythms: novel risk factors for obesity. Curr Opin Endocrinol Diabetes Obes 2016;23(05):353-359

24 Weinberger N-A, Kersting A, Riedel-Heller SG, Luck-Sikorski C. Body dissatisfaction in individuals with obesity compared to normal-weight individuals: a systematic review and meta-analysis. Obes Facts 2016;9(06):424-441

25 Mellor R, Chua SC, Boyce P. Antenatal depression: an artefact of sleep disturbance? Arch Women Ment Health 2014;17(04):291-302

26 LaGrotte C, Fernandez-Mendoza J, Calhoun SL, Liao D, Bixler EO, Vgontzas AN. The relative association of obstructive sleep apnea, obesity and excessive daytime sleepiness with incident depression: a longitudinal, population-based study. Int J Obes 2016;40 (09):1397-1404 